ARTICLE

\title{
Three body photodissociation of the water molecule and its implications for prebiotic oxygen production
}

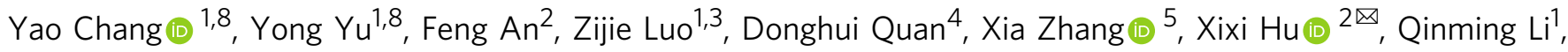
Jiayue Yang ${ }^{1}$, Zhichao Chen', Li Che ${ }^{3}$, Weiqing Zhang', Guorong Wu (D) 1, Daiqian Xie (1) 2,

Michael N. R. Ashfold (1) ${ }^{6}$, Kaijun Yuan (1) ${ }^{1 凶} \&$ Xueming Yang (1) ${ }^{1,7 凶}$

The provenance of oxygen on the Earth and other planets in the Solar System is a fundamental issue. It has been widely accepted that the only prebiotic pathway to produce oxygen in the Earth's primitive atmosphere was via vacuum ultraviolet (VUV) photodissociation of $\mathrm{CO}_{2}$ and subsequent two $\mathrm{O}$ atom recombination. Here, we provide experimental evidence of three-body dissociation (TBD) of $\mathrm{H}_{2} \mathrm{O}$ to produce $\mathrm{O}$ atoms in both ${ }^{1} \mathrm{D}$ and ${ }^{3} \mathrm{P}$ states upon VUV excitation using a tunable VUV free electron laser. Experimental results show that the TBD is the dominant pathway in the VUV $\mathrm{H}_{2} \mathrm{O}$ photochemistry at wavelengths between 90 and $107.4 \mathrm{~nm}$. The relative abundance of water in the interstellar space with its exposure to the intense VUV radiation suggests that the TBD of $\mathrm{H}_{2} \mathrm{O}$ and subsequent $\mathrm{O}$ atom recombination should be an important prebiotic $\mathrm{O}_{2}$-production, which may need to be incorporated into interstellar photochemical models.

\footnotetext{
${ }^{1}$ State Key Laboratory of Molecular Reaction Dynamics and Dalian Coherent Light Source, Dalian Institute of Chemical Physics, Chinese Academy of Sciences, Dalian, China. ${ }^{2}$ Key Laboratory of Mesoscopic Chemistry, School of Chemistry and Chemical Engineering, Institute of Theoretical and Computational Chemistry, Nanjing University, Nanjing, China. ${ }^{3}$ Department of Physics, School of Science, Dalian Maritime University, Dalian, Liaoning, China. ${ }^{4}$ Eastern Kentucky University, Richmond, KY, USA. ${ }^{5}$ Xinjiang Astronomical Observatory, Chinese Academy of Sciences, Urumqi, China. ${ }^{6}$ School of Chemistry, University of Bristol, Bristol, UK. ${ }^{7}$ Department of Chemistry, College of Science, Southern University of Science and Technology, Shenzhen, China.

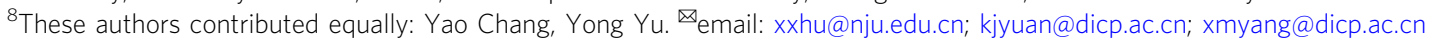


O xygen is the third most abundant element in the Universe, but its molecular form $\left(\mathrm{O}_{2}\right)$ is very rare. Besides on the Earth, molecular oxygen has only been detected in two interstellar clouds ${ }^{1,2}$, in the moons of Jupiter ${ }^{3}$ and Saturn ${ }^{4}$, and on Mars ${ }^{5}$. Geologically based arguments suggested that Earth's original atmosphere had no oxygen and was composed mostly of $\mathrm{H}_{2} \mathrm{O}, \mathrm{CO}_{2}$, and $\mathrm{N}_{2}$, with only small amounts of $\mathrm{CO}$ and $\mathrm{H}_{2}{ }^{6}$. Therefore, how oxygen is produced in the primitive atmosphere is a fundamentally important issue in the evolution of the early primitive atmosphere. Before the emergence of the oxygenrich atmosphere due to the "great oxidation event"7,8, about 2.33 billion years ago, which allowed the Earth to evolve into a living planet, a small amount of oxygen was already present, and this was previously attributed to an abiotic formation mechanism involving photodissociation of $\mathrm{CO}_{2}$ by vacuum ultraviolet (VUV) light, followed by three-body recombination processes ${ }^{9}$ :

$$
\begin{gathered}
\mathrm{CO}_{2}+h v \rightarrow \mathrm{CO}+\mathrm{O} \\
\mathrm{O}+\mathrm{O}+\mathrm{M} \rightarrow \mathrm{O}_{2}+\mathrm{M}
\end{gathered}
$$

where $\mathrm{M}$ is a third body to carry off the excess energy in the reaction process. Direct $\mathrm{O}_{2}$ production pathways via VUV photodissociation of $\mathrm{CO}_{2}{ }^{10}$ and dissociative electron attachment to $\mathrm{CO}_{2}{ }^{11}$ have recently been identified. These findings provide new insights into the sources of $\mathrm{O}_{2}$ in Earth's early atmosphere.

In contrast, photodissociation of $\mathrm{H}_{2} \mathrm{O}$, one of the dominant oxygen carriers ${ }^{12}$, has long been assumed to proceed mainly to produce hydroxyl $(\mathrm{OH})$ and hydrogen $(\mathrm{H})$ atom primary products, and contribute limitedly to the $\mathrm{O}_{2}$ production ${ }^{9}$. Recently, abundant molecular $\mathrm{O}_{2}$ in the coma of comet $67 \mathrm{P} /$ Churyumov-Gerasimenko, which is dominated by $\mathrm{H}_{2} \mathrm{O}, \mathrm{CO}$, and $\mathrm{CO}_{2}$, has been detected ${ }^{13}$. Interestingly, a strong correlation between $\mathrm{O}_{2}$ and $\mathrm{H}_{2} \mathrm{O}$ has been identified, indicating the $\mathrm{O}_{2}$ formation is linked to $\mathrm{H}_{2} \mathrm{O}$ in the comet. One plausible explanation for the strong $\mathrm{O}_{2}-\mathrm{H}_{2} \mathrm{O}$ correlation would be that the $\mathrm{O}_{2}$ is produced by radiolysis or photolysis of water, or single collisions of energetic $\mathrm{H}_{2} \mathrm{O}^{+}$with surfaces ${ }^{14}$. However, the existing photochemical reaction mechanisms underestimate the $\mathrm{O}_{2}$ abundance ${ }^{13}$. Thus, the detailed $\mathrm{O}_{2}$ production mechanism in the coma of comets is still unclear.

The photodissociation of water has been the subject of many experimental studies, which have revealed fascinating dynamics arising from strongly coupled electronic states with strikingly different potential energy surfaces (PESs) ${ }^{15,16}$. Excitation to the first excited singlet $\left(\tilde{A}^{1} B_{1}\right)$ state of $\mathrm{H}_{2} \mathrm{O}$ at wavelengths $\lambda \sim 160 \mathrm{~nm}$ results in direct $\mathrm{O}-\mathrm{H}$ bond fission yielding an $\mathrm{H}$ atom plus a ground state hydroxyl radical, $\mathrm{OH}\left(\mathrm{X}^{2} \Pi\right)$, with little internal excitation ${ }^{17-19}$. The absorption cross-section to the second excited singlet $\left(\tilde{B}^{1} A_{1}\right)$ state is maximal at $\lambda \sim 128 \mathrm{~nm}$. Excitation at these wavelengths results in a (minor) direct dissociation channel to electronically excited $\mathrm{OH}\left(A^{2} \Sigma^{+}\right)+\mathrm{H}$ products. The major dissociation process yields ground state $\mathrm{OH}(X)+\mathrm{H}$ products following non-adiabatic transitions at conical intersections between the $\tilde{B}$ and $\tilde{X}$ state PESs at linear $\mathrm{H}-\mathrm{O}-\mathrm{H}$ and $\mathrm{H}-\mathrm{H}-\mathrm{O}$ geometries ${ }^{20-23}$.

Additional fragmentation pathways, named three-body dissociation (TBD), become accessible energetically at shorter photolysis wavelengths, e.g.:

$$
\begin{gathered}
\mathrm{H}_{2} \mathrm{O}+h v \rightarrow \mathrm{O}\left({ }^{3} \mathrm{P}\right)+\mathrm{H}+\mathrm{H} \rightarrow(9.513 \mathrm{eV}) \\
\rightarrow \mathrm{O}\left({ }^{1} \mathrm{D}\right)+\mathrm{H}+\mathrm{H} \rightarrow(11.480 \mathrm{eV})
\end{gathered}
$$

where the threshold energies $\left(E_{\mathrm{th}}\right)$ for these fragmentation channels are given in parentheses (on the basis of thermodynamic calculations with the data available from the thermochemical network) (https://atct.anl.gov). Fragmentation channel (3) has been previously detected with small quantum yields ${ }^{24,25}$ following photoexcitation of $\mathrm{H}_{2} \mathrm{O}$ at the Lyman- $\alpha$ wavelength $(\lambda=121.57$ $\mathrm{nm})$. Because of the lack of intense tunable VUV laser sources, the quantitative assessment of the importance of the $\mathrm{H}_{2} \mathrm{O}$ TBD processes in the VUV region and its role in the $\mathrm{O}_{2}$ formation in the interstellar space has not been possible. Recent development of the intense VUV free electron laser, at the Dalian Coherent Light Source (DCLS), has provided an exciting tool for experimental studies of molecular photochemistry throughout the entire VUV region ${ }^{26}$.

Herein we report the studies of the $\mathrm{H}_{2} \mathrm{O}$ photochemistry in the VUV region using the DCLS and the $\mathrm{H}$-atom Rydberg tagging time-of-flight (HRTOF) technique. These experiments allow quantitative determination of the relative importance of the binary dissociation and the TBD processes following photoexcitation of $\mathrm{H}_{2} \mathrm{O}$ in the $90-110 \mathrm{~nm}$ region. The present results show conclusively that the $\mathrm{H}_{2} \mathrm{O}$ TBD process is an important pathway to form oxygen in the interstellar space.

\section{Results and discussion}

The $\mathrm{H}_{2} \mathrm{O}$ sample was generated in a supersonic beam, with a rotational temperature estimated to be about $10 \mathrm{~K}$. The $\mathrm{H}_{2} \mathrm{O}$ molecules were photoexcited to different Rydberg states ${ }^{27}$ (see Supplementary Fig. 2 and Supplementary Note 2). The dissociated $\mathrm{H}$ atom fragments were then detected using the HRTOF technique (see Supplementary Fig. 1 and Supplementary Note 1). TOF spectra of the $\mathrm{H}$ atoms resulting from $\mathrm{H}_{2} \mathrm{O}$ photodissociation at $\lambda=107.4 \mathrm{~nm}$ have been recorded, with the detection axis aligned parallel and perpendicular to the polarization vector of the VUV-FEL radiation. Knowing both the distance traveled by the $\mathrm{H}$ atom from the photodissociation area to the detector and its mass, the TOF spectra can be converted into the distributions of total kinetic energy release (TKER), which is derived using the following equation, $E_{\mathrm{KE}}=\frac{1}{2} m_{\mathrm{H}}\left(1+\frac{m_{\mathrm{H}}}{m_{\mathrm{OH}}}\right)\left(\frac{d}{t}\right)^{2}$, where $d$ is the flying path length of $\mathrm{H}$ atom $(d \approx 28 \mathrm{~cm})$ from the photodissociation region to the detector, $t$ is the measured time of flight ${ }^{28}$. Using the TKER distributions obtained in the parallel and perpendicular directions, we can construct a 3-dimensional (3D) flux diagram of the $\mathrm{H}$ atom fragments. Figure 1 shows the $3 \mathrm{D}$ product flux diagrams in two regions of the kinetic energy with rich structures. The tall feature at low kinetic energy (Fig. 1A) shows a large product angular anisotropy, whereas the product anisotropy in the higher kinetic energy region (Fig. 1B) is rather small.

For detailed analysis and feature assignment, the TKER distributions in parallel and perpendicular directions at $\lambda=107.4$ $\mathrm{nm}$ are plotted in Fig. 1C, while the product TKER distribution from photodissociation of $\mathrm{H}_{2} \mathrm{O}$ at the magic angle (with detection angle of $54.7^{\circ}$ relative to the polarization direction) is shown in Fig. 1D. These distributions show both sharp and broad features. Using the energy conservation relationship appropriate for a binary photodissociation process:

$$
E_{h v}+E_{\text {int }}\left(\mathrm{H}_{2} \mathrm{O}\right)=D_{0}(\mathrm{H}-\mathrm{OH})+E_{\mathrm{KE}}(\mathrm{H}+\mathrm{OH})+E_{\text {int }}(\mathrm{OH})
$$

where $E_{\text {int }}\left(\mathrm{H}_{2} \mathrm{O}\right)$ and $E_{\text {int }}(\mathrm{OH})$ are the internal energies of $\mathrm{H}_{2} \mathrm{O}$ and $\mathrm{OH}$, respectively, $E_{\mathrm{KE}}$ is the product total kinetic energy and $D_{0}(\mathrm{H}$ $-\mathrm{OH})$ is the dissociation energy of $\mathrm{H}_{2} \mathrm{O}^{29}$. We can assign all of the sharp structures to specific ro-vibrational levels of the $\mathrm{OH}$ product in the $\mathrm{X}$ and $\mathrm{A}$ states formed via the binary dissociation channel, $\mathrm{H}+\mathrm{OH}(\mathrm{X}$ or $\mathrm{A}, v, N)$. In addition to these sharp structures, the TKER spectra show two broad features: one with $E_{\mathrm{KE}} \leq 600 \mathrm{~cm}^{-1}$ that has a large angular anisotropy, and an underlying feature that spans the range of $600 \leq E_{\mathrm{KE}} \leq 16,000 \mathrm{~cm}^{-1}$ which displays a much smaller angular anisotropy. These broad features are obviously not from the binary dissociation channel, $\mathrm{H}+\mathrm{OH}$. Based on energy conservation, the maximum kinetic energy for the $\mathrm{H}$ atom product 


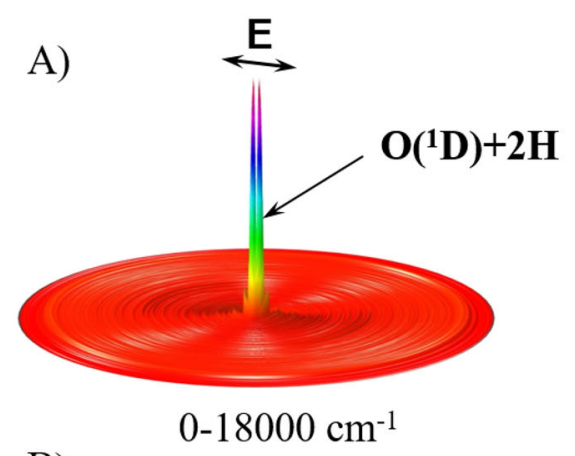

B)

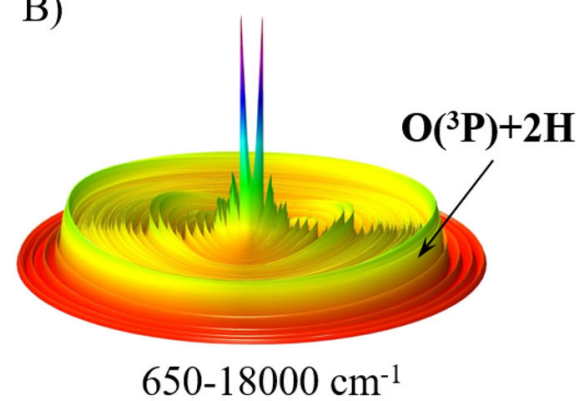

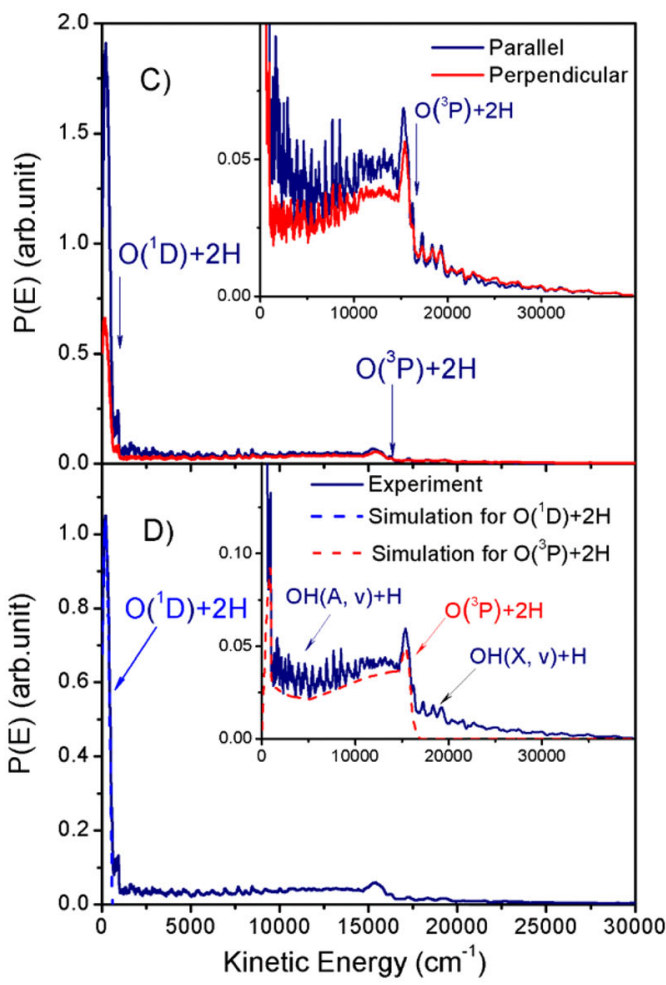

Fig. 1 The kinetic energy release spectra from $\mathbf{H}_{\mathbf{2}} \mathbf{O}$ photodissociation. A The $3 \mathrm{D}$ product contour diagram from the photodissociation of $\mathrm{H}_{2} \mathrm{O}$ at $107.4 \mathrm{~nm}$ for the total kinetic energy release (TKER) between 0 and $18,000 \mathrm{~cm}^{-1}$; B the 3D product contour diagram for the TKER between 650 and $18,000 \mathrm{~cm}^{-1}$. C The product TKER distributions at $\lambda=107.4 \mathrm{~nm}$ with the detection axis parallel and perpendicular to the polarization vector of the VUV-FEL radiation. The energetic limits of the two TBD channels $\left(E_{K E \max } \sim 580\right.$ and $\sim 16,448 \mathrm{~cm}^{-1}$ ) are marked, and the inset displays the same spectra on an expanded vertical scale. D The product TKER distribution at $\lambda=107.4 \mathrm{~nm}$ with the detection axis at $54.7^{\circ}$ (magic angle) to the polarization direction, along with the simulated TKER distributions for the $\mathrm{O}\left({ }^{1} \mathrm{D}\right)+2 \mathrm{H}$ and $\mathrm{O}\left({ }^{3} \mathrm{P}\right)+2 \mathrm{H}$ TBD channels. Source data are provided as a Source Data file.

from the TBD channels (3) and (4) at $107.4 \mathrm{~nm}$ are 16,448 and $580 \mathrm{~cm}^{-1}$, respectively. These limits of the two TBD channels match well with the upper limits of the two broad features in the distributions (Fig. 1). Thus, the intense broad feature at $E_{\mathrm{KE}}<600$ $\mathrm{cm}^{-1}$ is assigned to the $\mathrm{O}\left({ }^{1} \mathrm{D}\right)+2 \mathrm{H}$ channel, while the broad underlying signal extending to $E_{\mathrm{KE}} \sim 16,000 \mathrm{~cm}^{-1}$ is attributed to the $\mathrm{O}\left({ }^{3} \mathrm{P}\right)+2 \mathrm{H}$ products.

Given the above analysis, the product TKER distribution in Fig. 1D can be divided into three components: $\mathrm{O}\left({ }^{1} \mathrm{D}\right)+2 \mathrm{H}, \mathrm{O}\left({ }^{3} \mathrm{P}\right)+2 \mathrm{H}$, and $\mathrm{OH}+\mathrm{H}$. The first two components are broad features, while the third comprises sharp structures attributable to ro-vibrational levels of the $\mathrm{OH}(\mathrm{A})$ and $\mathrm{OH}(\mathrm{X})$ products. From Fig. $1 \mathrm{D}$, the $\mathrm{O}\left({ }^{1} \mathrm{D}\right)+2 \mathrm{H}$ channel obviously has the highest intensity in the kinetic energy $<600$ $\mathrm{cm}^{-1}$, yet it has never been reported previously in $\mathrm{H}_{2} \mathrm{O}$ photodissociation. The $\mathrm{O}\left({ }^{3} \mathrm{P}\right)+2 \mathrm{H}$ channel clearly makes the major contribution in the higher kinetic energy region. This indicates that the TBD is likely the dominant process following VUV photoexcitation of $\mathrm{H}_{2} \mathrm{O}$ at $107.4 \mathrm{~nm}$.

Branching ratios for the $\mathrm{O}\left({ }^{1} \mathrm{D}\right)+2 \mathrm{H}, \mathrm{O}\left({ }^{3} \mathrm{P}\right)+2 \mathrm{H}$, and $\mathrm{OH}+$ $\mathrm{H}$ fragmentation channels have been estimated by simulating the TKER distribution (Fig. 1D) using the three components shown in Fig. 2. Integrating the areas under the respective distributions returns relative $\mathrm{H}$ atom yields for the three channels. Recognizing that two $\mathrm{H}$ atoms are formed in each TBD process, the relative $\mathrm{H}$ atom yields are used to determine the branching ratio, e.g. $67 \%$ at $107.4 \mathrm{~nm}$ for the TBD channels (Table 1).

Photodissociation of $\mathrm{H}_{2} \mathrm{O}$ has also been investigated at eight more VUV wavelengths between 92 and $109 \mathrm{~nm}$, and a similar data analysis procedure is applied at these photolysis wavelengths (Supplementary Fig. 3 and Supplementary Note 3). The branching ratios determined for the binary and TBD channels at each wavelength are listed in Table 1. At $109.0 \mathrm{~nm}$, only one TBD channel $(\mathrm{O}$ $\left.\left({ }^{3} \mathrm{P}\right)+2 \mathrm{H}\right)$ exists because the $\mathrm{O}\left({ }^{1} \mathrm{D}\right)+2 \mathrm{H}$ channel is energetically not accessible. The results mean that oxygen atoms $\left({ }^{1} \mathrm{D}\right.$ and $\left.{ }^{3} \mathrm{P}\right)$, not $\mathrm{OH}$ radicals, are the major oxygen-containing products from $\mathrm{H}_{2} \mathrm{O}$ photolysis at $\lambda<107.4 \mathrm{~nm}$, in striking contrast to the dominant binary photofragmentation (i.e. $\mathrm{H}+\mathrm{OH}$ ) behavior displayed by $\mathrm{H}_{2} \mathrm{O}$ photochemistry at longer VUV wavelength $s^{15}$.

The dissociation dynamics of the two TBD channels are also quite interesting. Since the two $\mathrm{H}$ atoms in the water molecule are equivalent, if they dissociate simultaneously it should yield a narrow $\mathrm{H}$ atom kinetic energy distribution, peaking at an $E_{\mathrm{EK}}$ value close to half of the available energy (Supplementary Figs. 5 and 6 and Supplementary Note 5). However, the observed distributions are much broader than the narrow distributions for a simultaneous concerted process, implying that both TBD processes are due to mostly a sequential dissociation mechanism. Possible dissociation routes for the two channels are illustrated in Fig. 3 (and more details in Supplementary Fig. 7 and Supplementary Note 6). The water molecule undergoes efficient nonadiabatic coupling from the initial excited $n d$ Rydberg states to the $\widetilde{D}$ state. Path 1 illustrates the possible direct dissociation route from the $\widetilde{D}$ state to form $\mathrm{O}\left({ }^{1} \mathrm{D}\right)+2 \mathrm{H}$ products. The overall anisotropy parameter of this channel is about 0.8 , according with a fast and direct dissociation process. Paths 2 and 3 illustrate plausible routes for the more complicated $\mathrm{O}\left({ }^{3} \mathrm{P}\right)+2 \mathrm{H}$ dissociation paths: from the $\widetilde{D}$ state to the $\widetilde{B}$ state and then to the ground state via the two CIs between the $\widetilde{B}$ and $\widetilde{X}$ state PESs, following the initial internal conversion from the excited $n d$ Rydberg states to the $\widetilde{D}$ state. The averaged anisotropy parameter of this channel 
is considerably smaller $(\sim 0.2)$. This also accords with the dissociation undergoing several internal conversions, and with the more scrambling that leads to small product anisotropy.

The conclusion that the TBD is the dominant decay process following excitation of $\mathrm{H}_{2} \mathrm{O}$ at these VUV wavelengths could have profound implications for our understanding of the source of oxygen production. For quantitative assessment, we have calculated the fragment-dependent photodissociation rate of $\mathrm{H}_{2} \mathrm{O}$ by using: $J_{\mathrm{H} 2 \mathrm{O}}=\int \Phi_{\lambda} \Gamma \sigma_{\lambda} d \lambda$, where $\Phi_{\lambda}$ is the solar photon flux, $\Gamma$ is the fragment quantum yield, and $\sigma_{\lambda}$ is the photodissociation cross-section $^{30}$. Figure 4 collects together the wavelength dependences of the solar photon flux in the early period ${ }^{31}$, the total photoabsorption cross-sections of the parent $\mathrm{H}_{2} \mathrm{O}$ molecule in the VUV region $(90-200 \mathrm{~nm})^{32}$ and the production yields of $\mathrm{O}$

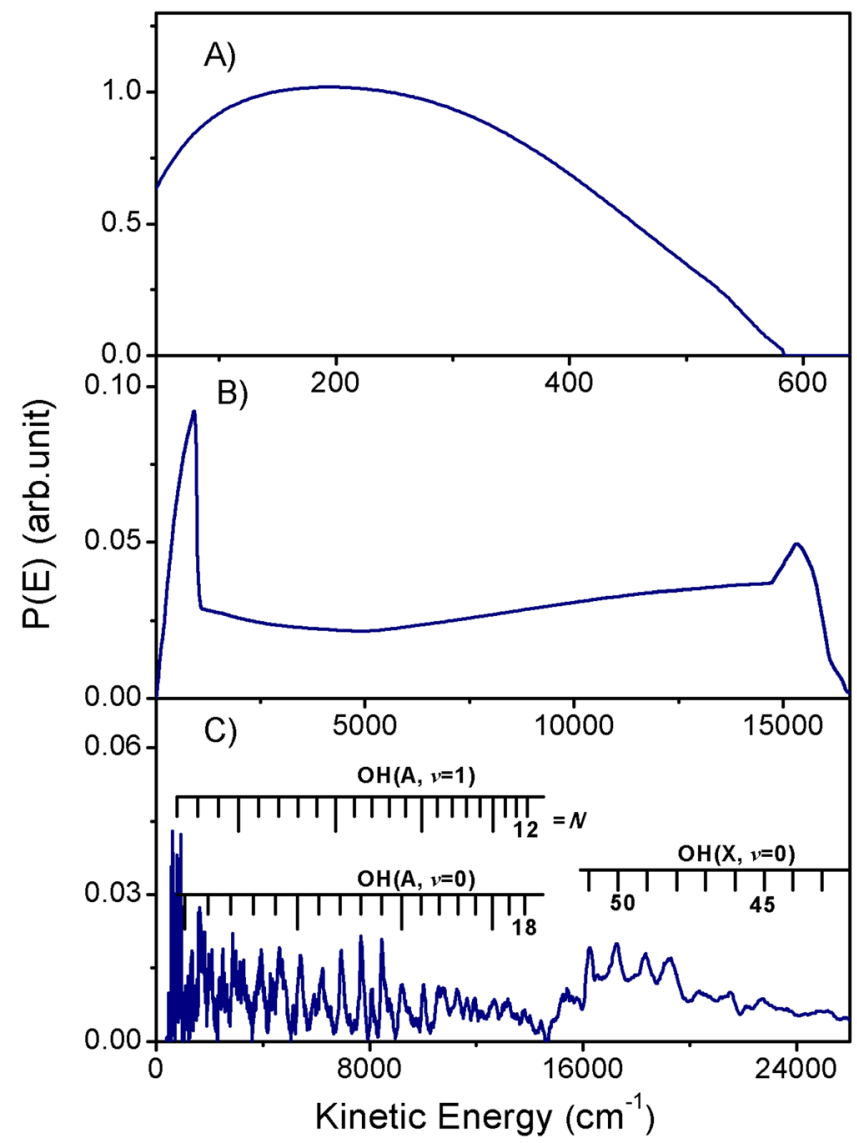

Fig. 2 The simulated kinetic energy release spectra from $\mathrm{H}_{2} \mathrm{O}$

photodissociation. TKER distributions determined from simulating the (A) $\mathrm{O}\left({ }^{1} \mathrm{D}\right)+2 \mathrm{H}$, (B) $\mathrm{O}\left({ }^{3} \mathrm{P}\right)+2 \mathrm{H}$, and (C) $\mathrm{OH}+\mathrm{H}$ product yields from photodissociation of $\mathrm{H}_{2} \mathrm{O}$ at $\lambda=107.4 \mathrm{~nm}$. The sharp features in the $\mathrm{H}+$ $\mathrm{OH}$ product yield have been assigned to population of ro-vibrational levels of the $\mathrm{X}$ and $\mathrm{A}$ states of the $\mathrm{OH}$ radical. Source data are provided as a Source Data file. atoms at studied photolysis wavelengths. Convoluting the solar photon flux, the photoabsorption cross-sections, and the production yields implies that $\sim 21 \%$ of the photoexcitation events of $\mathrm{H}_{2} \mathrm{O}$ will result in $\mathrm{O}$ atoms. Considering the water abundance in widely interstellar circumstances, like in interstellar clouds ${ }^{2,3}$ and in the moons of planets in the Solar System (e.g., the comets $67 \mathrm{P}^{13,33}$ ), oxygen production from the water photolysis should be an important process. The following recombination of oxygen atoms will produce molecular oxygen.

In addition, it is well known that the water photolysis has nothing to do with oxygen production in the Earth's atmosphere under equilibrium conditions due to VUV photon screening by the thick atmosphere ${ }^{10,34}$. However, in the earliest period of Earth, i.e., the period approaching to clement conditions on the earliest Earth followed by the current Earth-Moon system formed, the surface of Earth remained quite hot $(>1000 \mathrm{~K})^{34}$, all of the water on the Earth was vaporized to the atmosphere and part of water clouds (emitted from volcanos or delivered by carbonaceous chondrite meteorites ${ }^{6}$ ) populated at the top of the atmosphere could absorb the VUV photons and dissociate. Given $\left[\mathrm{H}_{2} \mathrm{O}\right]$ is ten times abundant than $\left[\mathrm{CO}_{2}\right]$ in the atmosphere during this early, chaotic period of Earth $^{6}$ (see Supplementary Fig. 4 and Supplementary Note 4), the O production rate from $\mathrm{H}_{2} \mathrm{O}$ VUV photochemistry could be three times larger than that of $\mathrm{CO}_{2}$ in the same VUV wavelength region, via TBD processes: $N_{\mathrm{H} 2 \mathrm{O}}(\mathrm{O}) / N_{\mathrm{CO} 2}(\mathrm{O})=\left(J_{\mathrm{H} 2 \mathrm{O}}(\mathrm{O}) \times\left[\mathrm{H}_{2} \mathrm{O}\right]\right) /\left(J_{\mathrm{CO} 2}(\mathrm{O}) \times\left[\mathrm{CO}_{2}\right]\right)=\sim 3$, where $J_{\mathrm{H} 2 \mathrm{O}}(\mathrm{O})=\sim 5.2 \times 10^{-5} \mathrm{~s}^{-1}$ (Fig. 4), $J_{\mathrm{CO} 2}(\mathrm{O})=\sim 1.8 \times 10^{-4}$ $\mathrm{s}^{-1}$ (Supplementary Fig. 8 and Supplementary Note 7), $\left[\mathrm{H}_{2} \mathrm{O}\right]$ and $\left[\mathrm{CO}_{2}\right]$ are the densities of $\mathrm{H}_{2} \mathrm{O}$ and $\mathrm{CO}_{2}$, respectively. Since the molecular oxygen generation process should be the same in the three-body recombination process (Eq. (2)), this analysis implies that the $\mathrm{H}_{2} \mathrm{O}$ photochemistry might be an important prebiotic source of $\mathrm{O}_{2}$ in Earth's early atmosphere.

From the experimental results, it seems that more than onethird of $\mathrm{O}$ atoms produced from $\mathrm{H}_{2} \mathrm{O}$ TBD process populate in the metastable ${ }^{1} \mathrm{D}$ state. The generation of $\mathrm{O}\left({ }^{1} \mathrm{D}\right)$ atoms from photodissociation of $\mathrm{H}_{2} \mathrm{O}$ in a significant amount is also very interesting because the metastable $\mathrm{O}\left({ }^{1} \mathrm{D}\right)$ atom is highly reactive ${ }^{35}$. It can react with almost all the gases emitted into the atmosphere. For instance, the reaction of $\mathrm{O}\left({ }^{1} \mathrm{D}\right)$ with methane could be a significant source of formaldehyde in the earth's primitive atmosphere ${ }^{36,37}$. Thus, the production of $\mathrm{O}\left({ }^{1} \mathrm{D}\right)$ atoms from the exposure of water to VUV radiation, and the subsequent reactions of these atoms, could have been important drivers in the evolution of the earliest atmosphere.

In the existing interstellar photochemical model, reactions (1) and (2) are the major pathways to produce prebiotic $\mathrm{O}_{2}$. In this work, we propose an alternative prebiotic $\mathrm{O}_{2}$ pathway: atomic oxygen production from the TBD of water, followed by oxygen recombination reactions. Recent International Ultraviolet Explorer satellite observation of pre-main-sequence stars suggested that the nascent Sun has emitted more than ten times VUV radiation than it does today ${ }^{38}$. This implies that oxygen formation by VUV photoinduced TBD of $\mathrm{H}_{2} \mathrm{O}$ is likely an

\begin{tabular}{|c|c|c|c|c|c|c|c|c|c|}
\hline \multirow[t]{2}{*}{ Dissociation channel } & \multicolumn{9}{|c|}{ Photolysis wavelength (nm) } \\
\hline & 109.0 & 107.4 & 106.7 & 105.7 & 101.3 & 98.1 & 96.2 & 94.5 & 92.0 \\
\hline$\overline{T B D}\left(\mathrm{O}\left({ }^{1} \mathrm{D} /{ }^{3} \mathrm{P}\right)+2 \mathrm{H}\right)$ & 0.35 & 0.67 & 0.76 & 0.62 & 0.77 & 0.72 & 0.79 & 0.86 & 0.86 \\
\hline Binary dissociation: $(\mathrm{H}+\mathrm{OH})$ & 0.65 & 0.33 & 0.24 & 0.38 & 0.23 & 0.28 & 0.21 & 0.14 & 0.14 \\
\hline
\end{tabular}




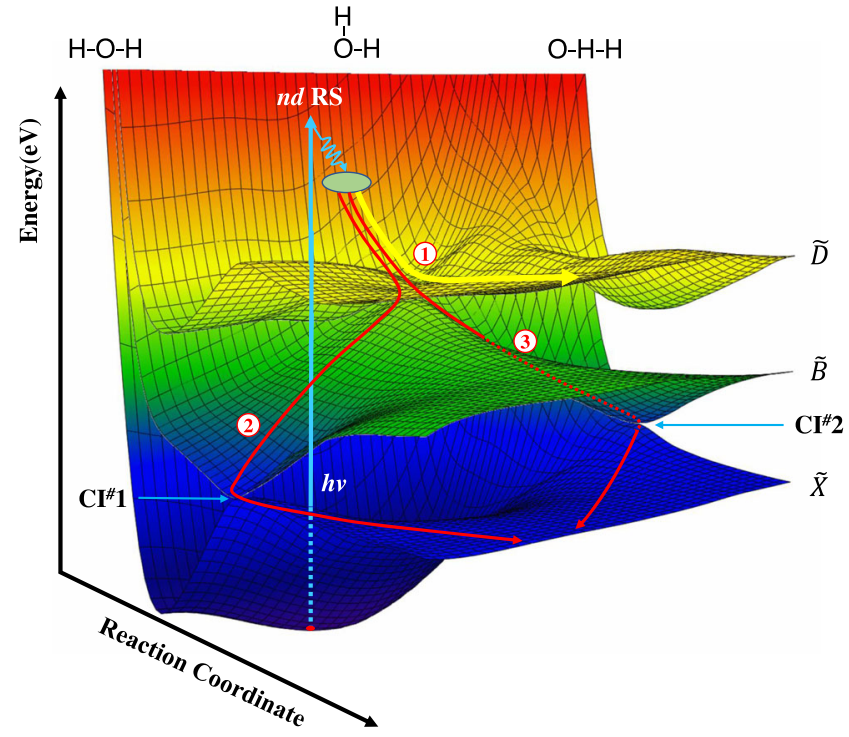

Fig. 3 Illustration of the TBD mechanisms of $\mathrm{H}_{2} \mathrm{O}$ upon VUV excitation. Photoexcitation populates the nd Rydberg state (nd RS) which undergo efficient non-adiabatic coupling to the $\widetilde{D}$ state. The light blue arrow displays the photoexcitation process and the gray oval represents the crossing region between the nd RS and the $\widetilde{D}$ state. Path 1 illustrates the possible direct dissociation route from the $\widetilde{D}$ state to form $\mathrm{O}\left({ }^{1} \mathrm{D}\right)+2 \mathrm{H}$ products (the yellow curve). Paths 2 and 3 illustrate plausible routes for the more complicated $\mathrm{O}\left({ }^{3} \mathrm{P}\right)+2 \mathrm{H}$ dissociation paths: from the $\widetilde{D}$ state to the $\widetilde{B}$ state and then to the ground state via the two conical intersections $(\mathrm{Cl} \# 1$ or $\mathrm{Cl}$ $\# 2$ ), following the initial internal conversion from the excited nd RS to the $\widetilde{D}$ state (the red curves). This picture is consistent with the observed angular anisotropy for the two channels: the $\mathrm{O}\left({ }^{1} \mathrm{D}\right)+2 \mathrm{H}$ channel is a more direct dissociation process with larger angular anisotropy, while the $\mathrm{O}\left({ }^{3} \mathrm{P}\right)+2 \mathrm{H}$ channel is a more complicated dissociation process with smaller angular anisotropy. The PES contour color from blue to red represents the potential energy from 0 to $12 \mathrm{eV}$.

important process in the coma of comets, in the interstellar clouds and even in Earth's primitive atmosphere, and thus needs to be incorporated into interstellar photochemical models. Furthermore, the TBD of $\mathrm{H}_{2} \mathrm{O}$ may well be important for the oxygen evolution in the atmospheres of all water-rich terrestrial planets ${ }^{39}$.

\section{Methods}

Vacuum ultraviolet free electron laser (VUV-FEL) radiation. The experiments employ a recently constructed apparatus for molecular photochemistry, which is centered on the VUV-FEL beam line at the DCLS ${ }^{26}$. The VUV-FEL facility runs in the high gain harmonic generation mode, in which the seed laser is injected to interact with the electron beam in the modulator (Supplementary Fig. 1). The seeding pulse, in the wavelength range $\left(\lambda_{\text {seed }}\right) 240-360 \mathrm{~nm}$, can be generated from a picosecond Ti:sapphire laser pulse. The electron beam is generated from a photocathode RF gun, and accelerated to the beam energy of $\sim 300 \mathrm{MeV}$ by seven $\mathrm{S}$ band accelerator structures, with a bunch charge of $500 \mathrm{pC}$. The micro-bunched beam is then sent through the radiator, which is tuned to the $2 \mathrm{nd} / 3 \mathrm{rd} / 4$ th harmonic of the seed wavelength, and coherent FEL radiation with wavelength $\lambda_{\text {seed }} / 2$, $\lambda_{\text {seed }} / 3$, or $\lambda_{\text {seed }} / 4$ is emitted. Optimization of the linear accelerator yields a high quality electron beam with emittance of $\sim 1.5 \mathrm{~mm}$ mrad, energy spread of $\sim 1 \%$, and pulse duration of $\sim 1.5 \mathrm{ps}$. In this work, the VUV-FEL operates at $10 \mathrm{~Hz}$, and the maximum pulse energy is $>100 \mu \mathrm{J} /$ pulse. The output wavelength is continuously tunable in the range $50-150 \mathrm{~nm}$ and the typical spectral bandwidth of the VUVFEL output is $30-50 \mathrm{~cm}^{-1}$.

The high- $n$ HRTOF technique used in this work was pioneered by Welge et al. ${ }^{40}$. The key point of this technique is the $1+1^{\prime}(\mathrm{VUV}+\mathrm{UV})$ excitation of the $\mathrm{H}$ atom. The first step involves VUV laser excitation of the $\mathrm{H}$ atom from its $n=1$ ground state to the $n=2$ state by absorbing one $\lambda=121.57 \mathrm{~nm}$ photon. In the second step, the $\mathrm{H}(n=2)$ atom is excited with a UV $(\lambda \sim 365 \mathrm{~nm})$ photon to a high- $n(n=30-80)$ Rydberg state. Charged species formed in the interaction
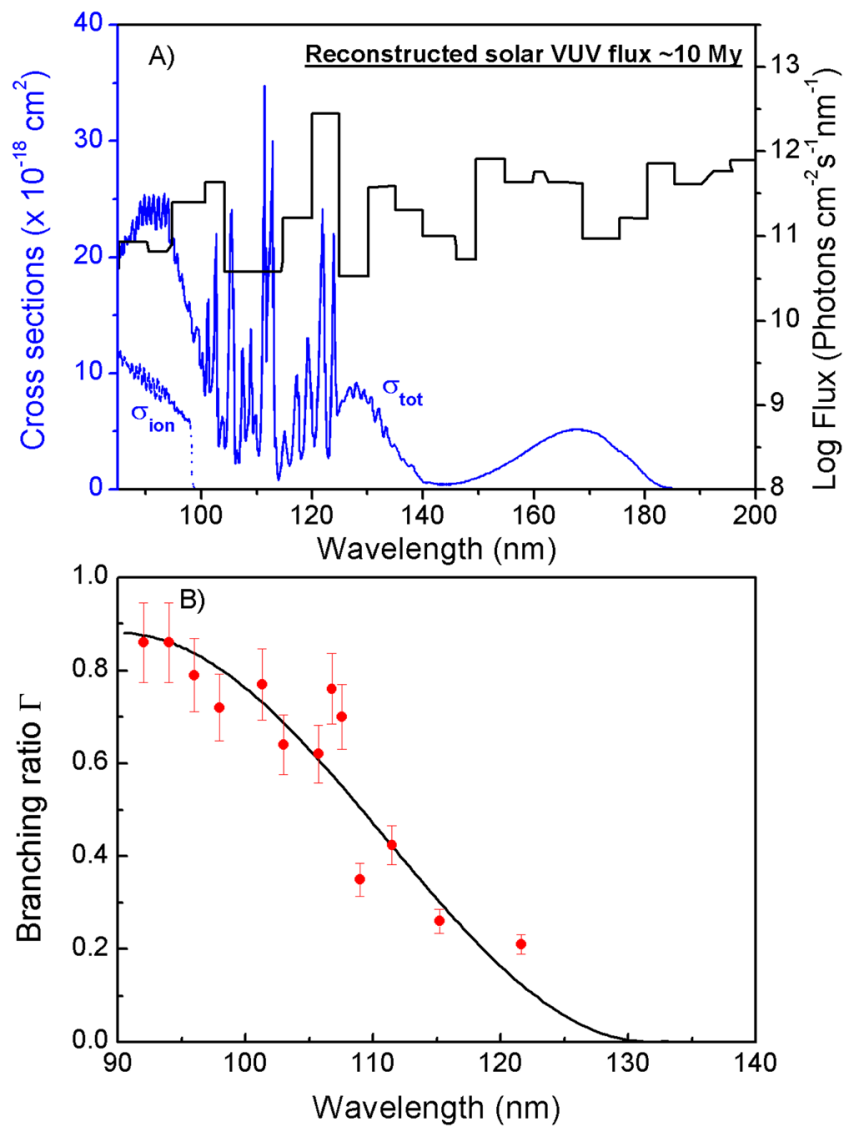

Fig. 4 The wavelength dependences of $\mathbf{O}$ atom quantum yields. Plot showing $(\mathbf{A})$ the wavelength dependences of the reconstructed VUV solar flux $\left(90-200 \mathrm{~nm}\right.$, the black lines) at $\sim 10 \mathrm{My}\left(10 \mathrm{My}=1 \times 10^{7}\right.$ years, reconstructed from ref. ${ }^{31}$. The VUV solar flux at modern period or the interstellar radiation field (ISRF) ${ }^{41}$ also can be used, which may modify the yield of O-production a little, but the final conclusion holds), the total absorption ( $\sigma_{\text {tot, }}$ the solid blue curve $)^{32}$ and photoionization ( $\sigma_{\text {ion }}$, the dotted blue curve) cross-sections 42 of $\mathrm{H}_{2} \mathrm{O}$, and (B) the quantum yield for forming O-atom photoproducts $\left(\mathrm{O}\left({ }^{3} \mathrm{P} /{ }^{1} \mathrm{D}\right)+2 \mathrm{H}\right), \Gamma$, determined in the present work (the red dot). It is noted that the predissociation rate of $\mathrm{H}_{2} \mathrm{O}$ is sufficiently fast that the fluorescence quantum yield must be negligible, so the total photodissociation cross-section will be almost the same as the photoabsorption cross-section. The polynomial function through the latter data is used to derive the reported overall $O$ product quantum yield (the black curve in B). The quantum yields at $\lambda=111.5,115.2,121.57 \mathrm{~nm}$ are obtained from refs. $15,24,26$. The error bars represent the standard deviation $(1 \sigma)$ of three times measurements. Source data are provided as a Source Data file.

region are extracted from the TOF axis by a small electric field $(\sim 20 \mathrm{~V} / \mathrm{cm})$ placed across this region. Rydberg tagged neutral $\mathrm{H}$ atoms fly a known distance $(d \approx 280$ $\mathrm{mm}$ ) from the interaction region to a rotatable microchannel plate (MCP) Z-stack detector located close behind a grounded fine metal grid. After passing through the grid, the Rydberg atoms are immediately field-ionized by the electric field ( $\sim 2000$ $\mathrm{V} / \mathrm{cm}$ ) applied between the grid and the front plate of the Z-stack MCP detector. The signal detected by the MCP is amplified by a fast preamplifier and counted by a multichannel scaler.

\section{Data availability}

All other data supporting this study are available from the authors upon request. Source data are provided with this paper.

Received: 14 December 2020; Accepted: 1 April 2021;

Published online: 30 April 2021 


\section{References}

1. Goldsmith, P. F. et al. Herschel measurements of molecular oxygen in Orion. Astrophys. J. 737, 96 (2011).

2. Liseau, R. et al. Multi-line detection of $\mathrm{O}_{2}$ toward $\rho$ Ophiuchi A. Astron. Astrophys. 541, A73 (2012).

3. Hall, D. T. et al. Detection of an oxygen atmosphere on Jupiter's moon Europa. Nature 373, 677-679 (1995).

4. Johnson, R. E. et al. Production, ionization and redistribution of $\mathrm{O}_{2}$ in Saturn's ring atmosphere. Icarus 180, 393-402 (2006)

5. Barker, E. S. Detection of molecular oxygen in the martian atmosphere. Nature 238, 447-448 (1972).

6. Zahnle, K., Schaefer, L. \& Fegley, B. Earth's earliest atmospheres. Cold Spring Harb. Perspect. Biol. 2, a004895 (2010).

7. Luo, G. M. et al. Rapid oxygenation of Earth's atmosphere 2.33 billion years ago. Sci. Adv. 2, e1600134 (2016)

8. Holland, H. D. The oxygenation of the atmosphere and oceans. Philos. T. R. Soc. B 361, 903-915 (2006).

9. Kasting, J. F., Liu, S. C. \& Donahue, T. M. Oxygen levels in the prebiological atmosphere. J. Geophys. Res. Oc. Atm. 84, 3097-3107 (1979).

10. Lu, Z., Chang, Y. C., Yin, Q. Z., Ng, C. Y. \& Jackson, W. M. Evidence for direct molecular oxygen production in $\mathrm{CO}_{2}$ photodissociation. Science 346, 61-64 (2014).

11. Wang, X. D., Gao, X. F., Xuan, C. J. \& Tian, S. X. Dissociative electron attachment to $\mathrm{CO}_{2}$ produces molecular oxygen. Nat. Chem. 8, 258-263 (2016).

12. Young, E. D. Strange water in the solar system. Science 317, 211-212 (2007).

13. Bieler, A. et al. Abundant molecular oxygen in the coma of comet 67P/ Churyumov-Gerasimenko. Nature 526, 678-681 (2015).

14. Yao, Y. X. \& Giapis, K. P. Dynamic molecular oxygen production in cometary comae. Nat. Commun. 8, 15298 (2017).

15. Yuan, K. J., Dixon, R. N. \& Yang, X. M. Photochemistry of the water molecule: adiabatic versus nonadiabatic dynamics. Acc. Chem. Res. 44, 369-378 (2011).

16. Yuan, K. J. et al. Nonadiabatic dissociation dynamics in $\mathrm{H}_{2} \mathrm{O}$ : competition between rotationally and nonrotationally mediated pathways. Proc. Natl Acad. Sci. USA 105, 19148-19153 (2008).

17. Engel, V., Schinke, R. \& Staemmler, V. Photodissociation dynamics of $\mathrm{H}_{2} \mathrm{O}$ and $\mathrm{D}_{2} \mathrm{O}$ in the 1 st absorption-band-a complete ab initio treatment. J. Chem. Phys. 88, 129-148 (1988).

18. Brouard, M., Langford, S. R. \& Manolopoulos, D. E. New trends in the stateto-state photodissociation dynamics of $\mathrm{H}_{2} \mathrm{O}($ A). J. Chem. Phys. 101, 7458-7467 (1994).

19. Yang, X. F., Hwang, D. W., Lin, J. J. \& Yang, X. Dissociation dynamics of the water molecule on the $\mathrm{A}^{1} \mathrm{~B}_{1}$ electronic surface. J. Chem. Phys. 113, 10597-10604 (2000).

20. van Harrevelt, R. \& van Hemert, M. C. Photodissociation of water. I. electronic structure calculations for the excited states. J. Chem. Phys. 112, 5777-5786 (2000).

21. Dixon, R. N. et al. Chemical "double slits": dynamical interference of photodissociation pathways in water. Science 285, 1249-1253 (1999).

22. Fillion, J. H. et al. Photodissociation of $\mathrm{H}_{2} \mathrm{O}$ and $\mathrm{D}_{2} \mathrm{O}$ in $\mathrm{B}, \mathrm{C}$, and $\mathrm{D}$ states (134-119 nm). Comparison between experiment and ab initio calculations. J. Phys. Chem. A 105, 11414-11424 (2001).

23. Mordaunt, D. H., Ashfold, M. N. R. \& Dixon, R. N. Dissociation dynamics of $\mathrm{H}_{2} \mathrm{O}\left(\mathrm{D}_{2} \mathrm{O}\right)$ following photoexcitation at the lyman-alpha wavelength (121.6 nm). J. Chem. Phys. 100, 7360-7375 (1994).

24. Harich, S. A. et al. Photodissociation of $\mathrm{H}_{2} \mathrm{O}$ at $121.6 \mathrm{~nm}$ : a state-to-state dynamical picture. J. Chem. Phys. 113, 10073-10090 (2000).

25. Slanger, T. G. \& Black, G. Photodissociative channels at $1216 \AA$ for $\mathrm{H}_{2} \mathrm{O}, \mathrm{NH}_{3}$, and $\mathrm{CH}_{4}$. J. Chem. Phys. 77, 2432-2437 (1982).

26. Wang, H. L. et al. Photodissociation dynamics of $\mathrm{H}_{2} \mathrm{O}$ at $111.5 \mathrm{~nm}$ by a vacuum ultraviolet free electron laser. J. Chem. Phys. 148, 124301 (2018)

27. Fillion, J. H. et al. High resolution photoabsorption and photofragment fluorescence spectroscopy of water between 10.9 and $12 \mathrm{eV}$. J. Chem. Phys. 120, 6531-6541 (2004).

28. Chang, Y. et al. Water photolysis and its contributions to the hydroxyl dayglow emissions in the atmospheres of Earth and Mars. J. Phys. Chem. Lett. 11, 9086-9092 (2020).

29. Boyarkin, O. V. et al. Accurate bond dissociation energy of water determined by triple-resonance vibrational spectroscopy and ab initio calculations. Chem. Phys. Lett. 568, 14-20 (2013)

30. Heays, A. N., Bosman, A. D. \& van Dishoeck, E. F. Photodissociation and photoionization of atoms and molecules of astrophysical interest. Astron. Astrophys. 602, A105 (2017).

31. Zahnle, K. J. \& Walker, J. C. G. The evolution of solar ultraviolet luminosity. Rev. Geophys. Space Phys. 20, 280-292 (1982).

32. Lee, L. C. \& Suto, M. Quantitative photoabsorption and fluorescence study of $\mathrm{H}_{2} \mathrm{O}$ and $\mathrm{D}_{2} \mathrm{O}$ at 50-190 nm. Chem. Phys. 110, 161-169 (1986).

33. Hassig, M. et al. Time variability and heterogeneity in the coma of 67P/ Churyumov-Gerasimenko. Science 347, aaa0276 (2015)

34. Sleep, N. H. The Hadean-Archaean environment. Cold Spring Harb. Perspec. Biol. 2, a002527 (2010)
35. Tachiev, G. I. \& Fischer, C. F. Breit-Pauli energy levels and transition rates for nitrogen-like and oxygen-like sequences. Astron. Astrophys. 385, 716-723 (2002)

36. Lin, J. J., Shu, J., Lee, Y. T. \& Yang, X. Multiple dynamical pathways in the O $\left({ }^{1} \mathrm{D}\right)+\mathrm{CH}_{4}$ reaction: a comprehensive crossed beam study. J. Chem. Phys. 113, 5287-5301 (2000).

37. Yung, Y. L. \& DeMore, W. B. Photochemistry of Planetary Atmospheres (Oxford University Press, 1999).

38. Zahnle, K. J., Gacesa, M. \& Catling, D. C. Strange messenger: a new history of hydrogen on Earth, as told by Xenon. Geochim. Cosmochim. Ac. 244, 56-85 (2019).

39. Zeng, L. \& Sasselov, D. The effect of temperature evolution on the interior structure of $\mathrm{H}_{2} \mathrm{O}$-rich planets. Astrophys. J. 784, 96 (2014).

40. Schnieder, L. et al. Photodissociation dynamics of $\mathrm{H}_{2} \mathrm{~S}$ at $121.6 \mathrm{~nm}$ and a determination of the potential energy function of $\mathrm{SH}\left(\mathrm{A}^{2} \Sigma^{+}\right)$. J. Chem. Phys. 92, 7027-7037 (1990)

41. van Dishoeck, E. F., Herbst, E. \& Neufeld, D. A. Interstellar water chemistry: from laboratory to observations. Chem. Rev. 113, 9043-9085 (2013).

42. Fillion, J. H. et al. Ionization yield and absorption spectra reveal superexcited Rydberg state relaxation processes in $\mathrm{H}_{2} \mathrm{O}$ and $\mathrm{D}_{2}$ O. J. Phys. B. Mol. Opt. Phys 36, 2767-2776 (2003)

\section{Acknowledgements}

The experimental work was supported by the National Natural Science Foundation of China (NSFC Center for Chemical Dynamics (Grant No. 21688102)), the National Natural Science Foundation of China (Grant Nos. 21873099, 21922306), the Strategic Priority Research Program of the Chinese Academy of Sciences (Grant No. XDB17000000), the Key Technology Team of the Chinese Academy of Sciences (Grant No. GJJSTD20190002), the Liaoning Revitalization Talents Program (XLYC1907154), and the international partnership program of Chinese Academy of Sciences (No. 121421KYSB20170012). The theoretical work was supported by the National Natural Science Foundation of China (Grant Nos 21733006, 22073042, and U1932147). M.N.R.A. is grateful for funding from the Engineering and Physical Sciences Research Council (EPSRC, EP/L005913) and to the NFSC Center for Chemical Dynamics for the award of a Visiting Fellowship.

\section{Author contributions}

K.Y. and X.Y. designed the experiments. Y.C., Y.Y., Z.L., Q.L., J.Y., and Z.C. performed the experiments. X.Z., D.Q., K.Y., M.N.R.A., L.C., W.Z., G.W., and X.Y. discussed the experimental results. F.A., X.H., and D.Q. performed the theoretical calculations. K.Y., M.N.R.A., and X.Y. prepared the manuscript.

\section{Competing interests}

The authors declare no competing interests.

\section{Additional information}

Supplementary information The online version contains supplementary material available at https://doi.org/10.1038/s41467-021-22824-7.

Correspondence and requests for materials should be addressed to X.H., K.Y. or X.Y.

Peer review information Nature Communications thanks Chaya Weeraratna, Piergiorgio Casavecchia and the other, anonymous, reviewer(s) for their contribution to the peer review of this work. Peer reviewer reports are available.

Reprints and permission information is available at http://www.nature.com/reprints

Publisher's note Springer Nature remains neutral with regard to jurisdictional claims in published maps and institutional affiliations.

Open Access This article is licensed under a Creative Commons Attribution 4.0 International License, which permits use, sharing, adaptation, distribution and reproduction in any medium or format, as long as you give appropriate credit to the original author(s) and the source, provide a link to the Creative Commons license, and indicate if changes were made. The images or other third party material in this article are included in the article's Creative Commons license, unles indicated otherwise in a credit line to the material. If material is not included in the article's Creative Commons license and your intended use is not permitted by statutory regulation or exceeds the permitted use, you will need to obtain permission directly from the copyright holder. To view a copy of this license, visit http://creativecommons.org/ licenses/by/4.0/

(C) The Author(s) 2021 